\title{
BRAND - the next generation receiver for VLBI
}

\section{W. Alef ${ }^{* a}$, G. Tuccari ${ }^{b a}$, S. Dornbusch ${ }^{a}$, M. Wunderlich ${ }^{a}$, M. Pantaleev ${ }^{c}$, J. Flygare ${ }^{c}$, J.D. Gallego ${ }^{d}$, J.A. López-Pérez ${ }^{d}$, F. Tercero ${ }^{d}$, G. Schoonderbeek ${ }^{e}$, J. Hargreaves ${ }^{e}$, R. de Wild ${ }^{e}$, V. Bezrukovs ${ }^{f}$}

${ }^{a}$ Max Planck Institute for Radio Astronomy

Auf dem Hügel 69, D-53121 Bonn, Germany

${ }^{b}$ INAF Istituto di Radioastronomia, Sezione di Noto

Contrada Renna, 96017 Noto (SR), Italy

${ }^{c}$ Onsala Space Observatory

SE-439 92 Onsala, Sweden

${ }^{d}$ Instituto Geográfico Nacional

Madrid, Spain

${ }^{e}$ ASTRON, Netherlands Institute for Radio Astronomy

Dwingeloo, The Netherlands

${ }^{f}$ Engineering Research Institute "Ventspils International Radio Astronomy Centre" of Ventspils

University of Applied Sciences, Inzenieru Street 101, Ventspils, LV-3601, Latvia

E-mail: walef@mpifr-bonn.mpg.de

The aim of the BRAND EVN project is to build a very wide receiver prototype for primary focus with a frequency range from $1.5 \mathrm{GHz}$ to $15.5 \mathrm{GHz}$ In addition we will investigate solutions for secondary focus telescopes. The project has received funding from the European Union's Horizon 2020 research and innovation programme under grant agreement No 730562 [RadioNet]. We present the status of the project which was started on January $1^{\text {st }}, 2017$ and is progressing smoothly: Feed, LNA, filters, major digital components, and some of the firmware are available now.

14th European VLBI Network Symposium \& Users Meeting (EVN 2018)

8-11 October 2018

Granada, Spain

*Speaker. 


\section{Introduction}

The BRAND EVN project will develop and build a prototype "digital" VLBI-receiver for the EVN (and other) telescopes. It will cover a frequency range of more than $1: 10$ from $1.5 \mathrm{GHz}$ to $15.5 \mathrm{GHz}$ ( $18 \mathrm{~cm}$ to $2 \mathrm{~cm}$ wavelength).

BRAND EVN is a so-called joint research activity (JRA) in RadioNet. It has received funding from the European Union's Horizon 2020 research and innovation programme under grant agreement No 730562. The budget is sponsored by the EU with about $1.5 \mathrm{M} €$ plus contributions by the partners: MPIfR, INAF/Noto, OSO, UAH/IGN, ASTRON, VUC ${ }^{1}$. The project started in January 2017 and it will end in June 2020. In case of need an extension by very few months is possible with the hard deadline of December 2020 when RadioNet ends.

This frequency band typically shows a lot of radio interference (RFI) which have to be eliminated from the data. Nevertheless we expect a large amount of bandwidth to be usable for VLBI. In addition this range includes the $2 \mathrm{~cm}$-band which has not been available on the $\mathrm{EVN}$, but is heavily used on the VLBA.

The BRAND receiver covers the standard EVN frequency bands which are in highest demand by the observers: $18 \mathrm{~cm}, 13 \mathrm{~cm}, 6 \mathrm{~cm}, 5 \mathrm{~cm}$ and $4 \mathrm{~cm}$ where not all EVN stations have all frequencies $^{2}$. The majority of these receivers are single frequency receivers, which typically have to be exchanged when a band change occurs in an observing session. A receiver change usually takes hours of manual labour. As a result, in the three EVN observing sessions per year between three and four frequency bands can be observed in succession. The important point is that multi-band (quasi-) simultaneous observations are not possible with the EVN.

The Very Large Baseline Array (VLBA) on the other hand offers fast frequency switching (on the order of seconds) which allows two or three frequencies to be observed quasi-simultaneously in a single observation. This has been a very successful mode of operation with high user demand.

BRAND receivers, once available on most EVN telescopes, will enable real multi-frequency VLBI without frequency switching. As BRAND will be one single receiver with a single LNA, a single sampler chip and a single backend, it will be possible to fringe-fit over the whole observed band coherently by fitting also for the quadratic delay over frequency without having to care for unknown phase offsets between the usable parts of the band. Developing such a fringe-fitting algorithm is one of the tasks of the RadioNet JRA RINGS. A similar fringe-fitter exists for the geodetic VGOS project(see e.g. [1]).

It was decided to engineer the prototype for primary focus, as wide-band feeds for it are much more advanced than feeds for secondary focus. (reference to VGOS) In the preparatory phase it was also decided to install the receiver on the Effelsberg $100 \mathrm{~m}$-telescope to maximise the visibility of the project.

As a number of telescopes in the EVN can only mount secondary focus receivers the project has a work package to do research on secondary focus feed which would work for the BRAND frequency range. The final deliverable for the WP will be a report on the results of the simulations of such a feed.

\footnotetext{
${ }^{1}$ See list of institutions on title page

${ }^{2}$ Additional receivers exit at $1.3 \mathrm{~cm}, 21 \mathrm{~cm}, 7 \mathrm{~mm}$, and $50 \mathrm{~cm}$ (in decreasing order of importance)
} 
The largest scientific impact of the BRAND receiver will be caused by enabling real multiwavelength VLBI. Together with the RINGS fringe-fitting this will allow precise relative registration of simultaneous images at different frequencies, because the visibility phases can be connected over the whole frequency range. This can in general not be achieved with fast frequency switching. At the same time the very large (usable) bandwidth over-compensates the reduced sensitivity of receiver components due to the ultra-wide bandwidth. The wide bandwidth will also improve the UV-coverage vastly as UV-coordinates vary as a function of frequency. So the ellipses caused by earth synthesis will become broad tracks in the UV-plane.

Other important scientific opportunities will arise from the compatibility with the VGOS network of antennas, such that telescope positions can be tied again to the geodetic network of antenna coordinates. Astronomical antennas can then also contribute to important astrometric projects like the ICRF3 catalogue (see e.g. [2]).

With multi-frequency VLBI in spectroscopic VLBI several different maser types can be observed in different frequency bands simultaneously, and absolute relative positions can be derived.

VLBI observations to determine Rotation Measures will also profit from the wide band and relatively small gaps between the bands. The same is true for some single-dish observations like flux density monitoring, pulsar search or polarisation observations.

\section{BRAND project description and status}

\subsection{BRAND overview}

The project team is made up of members of the partner institutions: MPIfR, INAF/Noto, OSO, IGN/UAH, ASTRON, and VUC ${ }^{3}$. The project structure can be seen in figure 1. WPs 6.2 to 6.5 are the logical WPs for building a "digital" receiver.

In WP6.1 relevant information of preferably all EVN antennas for the future installation of BRAND receivers was assembled by IGN/UAH. The resulting report was submitted in June 2017. The document though will be augmented during the project with all further relevant information and data.

The BRAND receiver will use an innovative scheme where the analogue signals will be converted into the digital domain close to the receiver (see figure 2). According to our receiver engineers the dynamic range of optical fibres for the transmission of analogue signals is at most about $30 \mathrm{~dB}$, which is not enough for the expected RFI remaining after initial filtering before the LNA. The data will be processed in a first digital stage right after sampling to select the bands which have good data or are wanted for a particular observation. The digital data is then transmitted to the backend room where the data is further split into sub-bands, and is formatted into VDIF packets by a DBBC3 for recording or transmission via Internet.

\subsection{Feed horn}

The feed horn was developed by OSO. The final solution found for Effelsberg is a QuadRidge Flared Horn feed (QRFH) with an additional dielectric inset which improves the efficiency of the feed at higher frequencies [3]. To find a satisfactory solution for Effelsberg was particularly

\footnotetext{
${ }^{3}$ See list of authors
} 


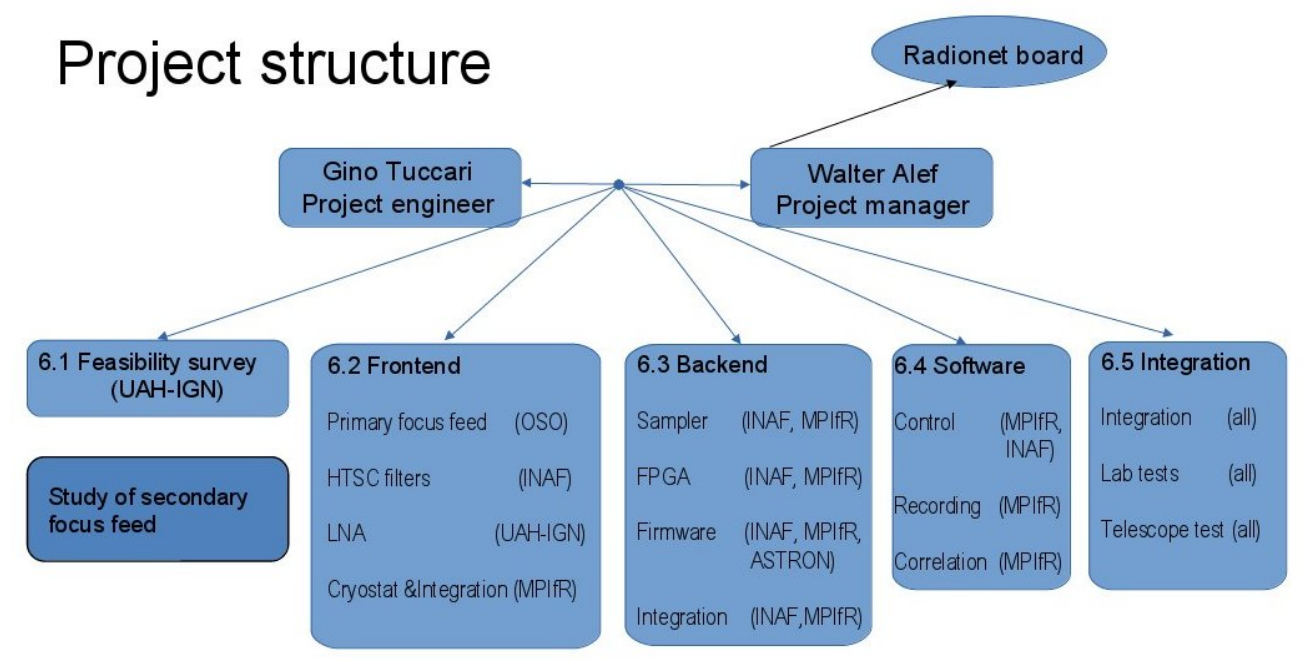

Figure 1: In addition to the Work Packages for the receiver prototype, there are a feasibility study to prepare the implementation of BRAND receivers in the EVN, and a study for a BRAND secondary focus feed.

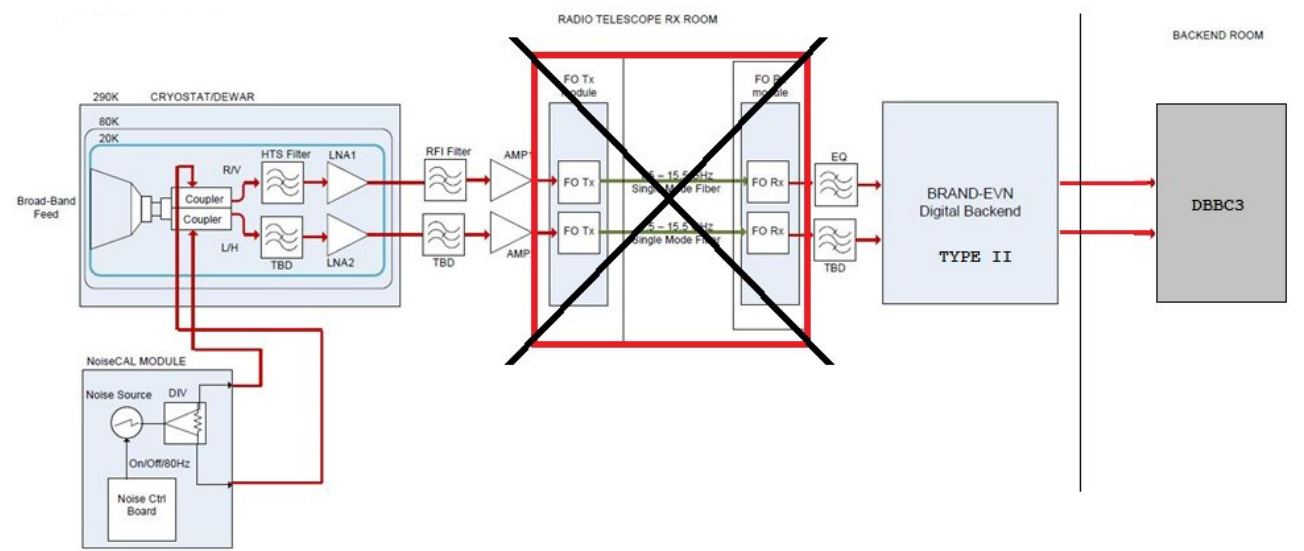

Figure 2: Block diagram of the BRAND receiver. Transmission of analogue signals to the backend room will be avoided due to dynamic range limitations of analogue signals over fibre. Sampling and first digital processing will be close to the receiver.

difficult due to the extreme opening angle of $2 \times 79^{\circ}$ and an $f / D=0.3$. The most important feed characteristics (over whole band) are an average aperture efficiency of $50 \%$ with an input reflection better than $-10 \mathrm{~dB}$ (see figure 3). The estimated sensitivity of the feed (SFED) in the Effelsberg dish is between $20 \mathrm{Jy}$ and $40 \mathrm{Jy}$ which is not much worse than for narrow band receivers.

The feed has been manufactured and delivered to OSO where laboratory measurements are ongoing.

\subsection{HTSC filters}

High Temperature Superconductor (HTSC) Filters are needed to suppress the strongest RFI which would drive the LNA and/or the sampler into the non-linear regime. The HTSC filters will 

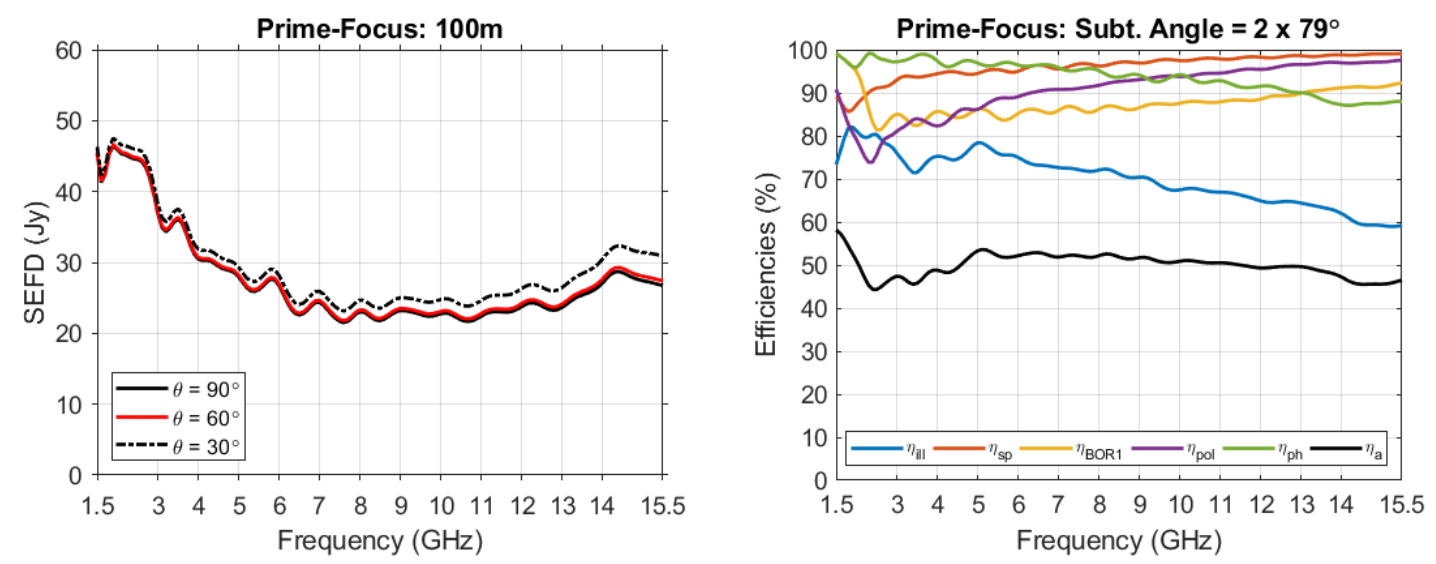

Figure 3: Left: Simulated sensitivity for Effelsberg 100 m. Right: Black line shows simulated efficiency.

be directly coupled to the feed. From the RFI survey done at Effelsberg as part of WP6.1 it was concluded that two notch filters will be needed at $1.8 \mathrm{GHz}$ and $2.2 \mathrm{GHz}$. In addition a high pass filter has to cut below $1.5 \mathrm{GHz}$, because there a lot of strong RFI exists and the feed does not sufficiently attenuate those frequencies. For calibration purposes a directional coupler for phase-cal and noise calibration is very desirable.

Simulations of a HTCS filter with the large BRAND bandwidth plus notches proved to be really difficult, and so far no optimal solution could be derived. Presently we are investigating compromises and alternatives. For instance we might use two filters attached to each other. Which option to choose will be decided soon, as the filters will be needed to integrate the feed with the LNA.

\subsection{Low Noise Amplifier}

Results of the IGN/UAH group showed that a single Low Noise Amplifier (LNA) has the lowest noise over the BRAND band. But the Input Return Loss (IRL) is unacceptably high at the low frequency end. The overall best solution for the extreme bandwidth found are balanced amplifiers which are made up of two additional hybrids and two LNAs which means higher cost and more complexity. The noise is slightly higher while the IRL is acceptable (see figure 4).

For BRAND it was decided to use a balanced amplifier. As this amplifier needs a hybrid before the two LNAs, it can be used to convert the feed linear polarisation to circular. Despite of this being a veritable challenge it will be attempted by the Yebes group.

\subsection{Sampler and FPGA processor}

The sampler chip has four inputs, and can sample $14 \mathrm{GHz}$ at a rate of $28 \mathrm{Gsps}$ or $16 \mathrm{GHz}$ at a rate of 56 Gsps. It is clear that the preferred mode should be to sample at a rate of $28 \mathrm{Gsps}$ as this is less demanding on the FPGA processor. So the two inputs for the two polarisations will be split in four bands, one from $1.5 \mathrm{GHz}$ to $14 \mathrm{GHz}$ and the second from $14 \mathrm{GHz}$ to $15.5 \mathrm{GHz}$.

Initially the sampler evaluation board will be used to gain experience with the requirements for the sampling board, the processor, and the firmware to receive and reconstruct the data in the FPGA processor. The FPGA selected is a Xilinx Kintex Ultrascale. 

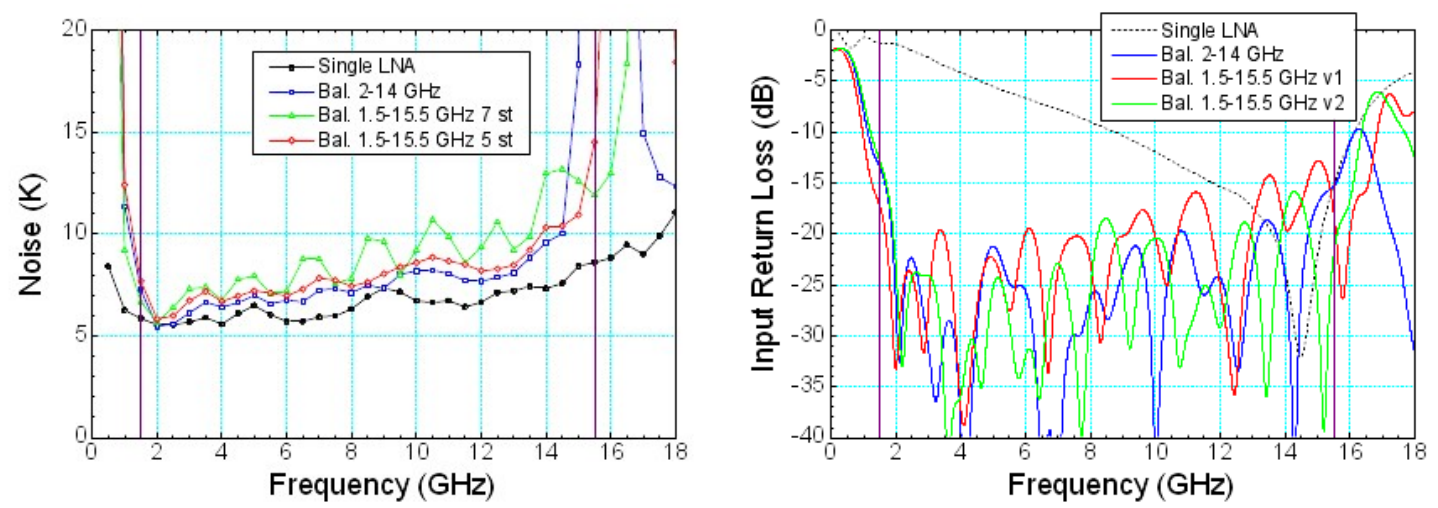

Figure 4: Left: Amplifier noise as a function of frequency. The single LNA has the lowest noise Right: IRL as a function of frequency. The single LNA shows a very high IRL at low frequencies.

The firmware is in different stages of development. The code for data reception and reconstruction is well under way. The firmware for band-selection and VLBI sub-bands is mostly ready, while code for transporting the data out over Ethernet has not yet been started. Work on the digital polarisation conversion will start at the end of the year. A total of more than two FTEs resulting in far more than 100,000 lines of VHDL code have been written so far.

\subsection{Software, testing, and secondary focus feed research}

The software to control the receiver will be derived from software used at Effelsberg, while the digital part will be an extension of the DBBC3 control software. Modifications for recording and correlation software will be started later in the project.

The integrated receiver will be tested in the laboratory and on the telescope in the first half of 2020. The final test will be a VLBI observation preferably with some VGOS antennas which have four simultaneous frequency bands of $0.5 \mathrm{GHz}$ of $1 \mathrm{GHz}$ in the range from about $3 \mathrm{GHz}$ to $14 \mathrm{GHz}$.

Research on a secondary focus feed for BRAND will start in the second half of 2018.

\section{Acknowledgments}

This work has received funding from the European Union's Horizon 2020 research and innovation programme under grant agreement No 730562 [RadioNet].

\section{References}

[1] A. Niell, J. Barrett, A. Burns, R. Cappallo, B. Corey, M. Derome, C. Eckert, P. Elosegui, and 15 others, Demonstration of a Broadband Very Long Baseline Interferometer System: A New Instrument for High-Precision Space Geodesy, Radio Science, 53, Issue 10, p. 1269-1291, October 2018.

[2] J. Frouard, M.C. Johnson, A. Fey, V.V. Makarov, B.N. Dorland, Toward the ICRF3: Astrometric Comparison of the USNO 2016A VLBI Solution with ICRF2 and Gaia DRI, AJ, 155, Issue 6, article id. 229,11 pp. (2018). 
[3] J. Flygare, M. Pantaleev, and S. Olvhammar, "BRAND: Ultra-Wideband Feed Development for the European VLBI Network - A Dielectrically Loaded Decade Bandwidth Quad-Ridge Flared Horn”, in: 12th European Conference on Antennas and Propagation (EuCAP2018), London, UK, 9-13 April 2018. 